\title{
Selected methods of artificial intelligence for Internet of Things conception
}

\author{
Aneta Poniszewska-Maranda \\ Lodz University of Technology \\ ul.Wolczanska 215, 90-924 Lodz, Poland \\ Email: aneta.poniszewska-maranda@p.lodz.pl
}

\author{
Daniel Kaczmarek \\ Lodz University of Technology \\ ul.Wolczanska 215, 90-924 Lodz, Poland \\ Email: dkdaniel@vp.pl
}

\begin{abstract}
The concept of Internet of Things appeared several years ago and in that time has evolved into one of pillars of the new technologies sector. The next step is to add the artificial intelligence to Internet of Things systems. Artificial intelligence is increasingly used in everyday life. It is a concept of a wide range and applies in practice in many fields of science. The aim of presented paper was to investigate the usefulness of selected artificial intelligence methods in the concept of Internet of Things. To investigate this purpose, exemplary system was built and it uses the artificial neural networks.
\end{abstract}

\section{INTRODUCTION}

$\mathbf{T}$ HE Internet is a powerful tool used in all kinds of the information systems. The network is available almost anywhere, at home, at work, also on mobile devices (phones, watches). People start to think to connect the Internet to almost all devices of everyday use, so they can communicate with each other by taking simple decisions for people and helping them in their life. Such idea is called the Internet of Things (IoT). It is estimated that currently about 15 billion devices are connected to the Internet, but this number is still less than $1 \%$ of things that in fact could be connected to the network [10].

The next step is to add the artificial intelligence to Internet of Things systems. Artificial intelligence is increasingly used in everyday life. It is a concept of a wide range and applies in practice to many fields of science. It is used in applications such as prompting videos to watch, having regard to the history of the watch (service netflix) or recognize people on the recordings of the monitoring. Its great advantage is the elements related to machine learning, through which different methods of artificial intelligence are able to interpret a lot of data and present some of their summary. This is definitely a big amenity for a man who no longer has to statically analyse all the data coming from the specified system, for example view a recording of the monitoring in the context of searching for a particular person.

The presented paper examines whether the chosen methods of artificial intelligence are suitable for use in the concept of Internet of Things. The main assumption is to use the mobile device (mobile phone) as a smart object in the Internet of Things. The system based on the concept of Internet of Things was designed and built to examine this issue and this system was then implemented with different methods of artificial intelligence.
The presented paper is structured as follows: section 2 presents the issues of Internet of Things concept with its definitions, section 3 gives the outline of the architecture of IoT, presenting the three-layer and five-layer architectures, while section 4 deals with the aspect of communication in IoT concept. Section 5 describes the use of selected methods of artificial intelligence in the conception of IoT and section 6 presents the created exemplary IoT system using such methods.

\section{CONCEPT OF INTERNET OF THINGS}

The concept of the Internet of Things appeared several years ago and in that time has evolved into one of the pillars of new technologies sector. There is no clear definition of this concept in the literature. In many cases, the definitions are complementary, creating a more accurate description of the problem.

The Internet of Things is a vision, in which objects become part of the Internet, where every object is uniquely identifiable and accessible on the Web. These objects may directly or indirectly collect, process or exchange data via data communications network. This concept can be described by a simplified equation [1]:

physical objects + sensors and microprocessors $=I o T$

One of the first attempts to define this concept was pretty simple concept. The Internet of Things is all objects in everyday life, which are equipped with wireless identifiers and so that they can communicate with each other and be managed by a computer [3].

The shortest definition, which may describe the concept of the Internet of Things shows that it is clearly identification of objects and their representation in the structure of the Internet. This is the most general definition that may expresses the most important elements concerning the issue of IoT [4].

By analysing this information, it can be concluded that the IoT systems have a lot of common issues with multi-agent systems. However, it is worth saying that these two concepts are not identical and have some subtle differences. One of the important difference is a fact that all objects in the concept of the Internet of Things should be able to communicate with the environment, be aware of what they are and be clearly identifiable in their environment [5]. 
On the basis of all above definitions of the issues of IoT and taking into account its important aspects, the concise and yet comprehensive definition of this concept was created. Thus,

\section{Internet of Things is a concept, where clearly identifiable and smart objects can communicate with each other in a defined environment to make autonomous decisions by analysing and processing the data collected from the environment.}

The environment can be the Internet or only a portion (e.g. local area network and the devices used only at home). Additionally, there may be mentioned also that the interconnected devices provide the user with various number of applications and services which enable it to communicate with them. To take the autonomous decisions, except to analyse the collected information, objects may use generally understood knowledge, that is, for example, elements related to the habit of the user in some aspect, so that the object will be able to make the better decisions.

\section{Architecture of Internet of Thing}

Architecture and technology for smart objects, included into Internet in accordance with the concept of Internet of Things must be extremely flexible. They have to provide the infrastructure, taking into account the heterogeneity of devices and the need for ubiquitous communication, which must be continuous. Just as in the definition of the concept of IoT, specifying its architecture can not clearly define how it should look like. There are many general proposals for IoT infrastructure, but not all of them are sufficiently flexible.

To start talking about specific aspects concerning system architecture based on the IoT, we need first to mention the main pillars of this concept, which will be the basis for the design of adequate infrastructure, namely (Fig. 1):

- anytime,

- anyplace,

- anything.

Taking into account the main pillars and intelligent objects features posted on the Internet, the main aspects that the IoT architecture must meet can be presented. This means for example, that every item included in the Internet of Things (considering its first feature) has a unique identifier in the particular environment in which it occurs and it is available anytime, anywhere and with anything (for all other devices in the structure). Similarly, we can develop two more features of intelligent objects in conjunction with the main pillars of the IoT. This analysis makes us aware that the provision of adequate infrastructure in accordance with the concept of the Internet of Things is not a trivial issue [1], [2], [6].

Implementation of systems based on the concept of Internet of Things requires a comprehensive look at the infrastructure, from the lowest layers of collecting data from the sensors to the highest layers presenting the user different statements of analysed data.

\section{A. 3-layer architecture}

The basic architecture which can be successfully used in such systems is called 3-layer architecture of IoT. It consists of the following layers, starting from the lowest [7], [8]:

- perception layer,

- transport layer (network layer),

- application layer.

Perception layer is the first layer in a three-tier architecture of IoT. It is responsible for collecting the data from the real world. Its role is to combine the real world with the virtual world in the context of collection and pre-processing of information. It includes various types of sensors or electronic tags. Most of the modern smartphones are equipped with various sensors, such as ambient light sensor, accelerometer, gyroscope and proximity sensor. This means that the mobile phone can be successfully used in the smart object of IoT, which additionally can pre-process the collected data so that they will be readable for the rest of the system [9], [7].

The second layer, transport layer (network), of this architecture provides the processing of data from sensors, some local storage and forwarding them to the application layer. This layer can be compared to a neural network located in the brain. Since it depends which way and where (to which device) the information will be provided. One of its main tasks is to ensure the effective reach of information and taking care of continuous operations. The network layer is based on modern communication technologies, wired and wireless.

The last layer of this architecture, application layer, is the most extensive. It ensures the delivery of services and applications for the end users. It may seem that the important element in this layer is to ensure a user-friendly interaction with the interface. However, this is a small part of the responsibility of the layer. Its fundamental role is to provide a platform for data collection and subsequent analysis. It is understood as its appropriate interpretation, processing and establishing the relationships.

\section{B. 5-layer architecture}

The 3-layer architecture is the fundamental architecture of the Internet of Things. It describes all the necessary infrastructure elements used in the IoT, however, by the fact that there are relatively few layers, each of which is responsible for many aspects. In consequence, it should be more extract components attributable to the particular layer. Such elements include a 5-layer architecture. Its use increases the flexibility of the entire infrastructure.

The 5-layer architecture of IoT introduces two additional layers between the existing layers of 3-layer architecture. Starting from the lowest layer, this architecture has the following (Fig. 2) [9]:

- perception layer,

- access to gateway layer,

- network layer,

- middleware layer,

- application layer. 


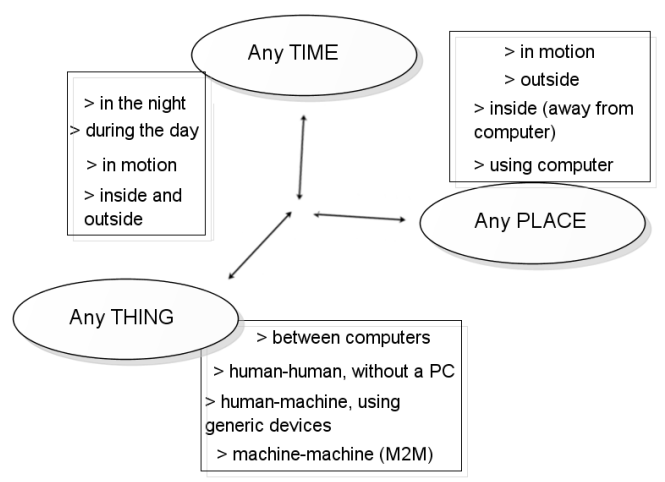

Fig. 1. Schema presenting the main pillars of IoT concept for its communication

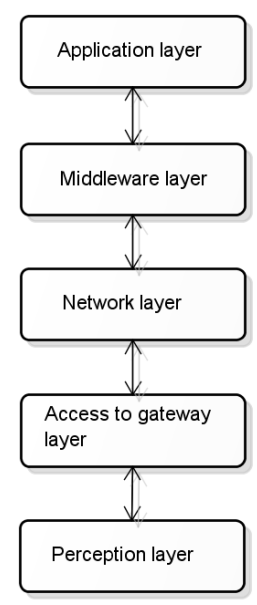

Fig. 2. Schema presenting the 5-layer architecture of IoT

The first layer, perception layer, just is primarily responsible for the collection of data from the environment. Another, a new access to gateway layer supports the communication network layer. Both these two layers are responsible for management of data in exchange infrastructure. The main idea of adding a new layer is to transfer the data directly between the intelligent objects.

With this approach, we can definitely increase the flexibility of the system due to the ability to isolate the communication between devices and services. This second layer is a communication bus between objects and network gateway.

Another newly-added layer is called middleware layer. The main purpose of this layer is to increase the flexibility of the interface between the hardware and the software. Information coming from network layer is transmitted to the middleware layer, which is a certain abstraction from both the upper and lower layers. It can be compared to API in the context of the issue of appropriate interfaces for application layer in data management. This approach makes it easier to make the modifications in the whole computer system [9].

The presented architectures form a whole and their mutual application (including joining) may successfully allow to build the complex systems based on the IoT.

\section{COMmunication Between InTERnet OF Things ELEMENTS}

Today, the concept of the Internet of Things is a component of many different technologies. From a logical point of view, the systems based on the issue of IoT can be seen as a set of smart devices that work together to achieve a set goal. From a technical point of view, such system may consist of several modules, each of which can be implemented in different technologies, uses different architecture and thus uses different items related to the processing of data and even the internal communication [1], [11].

The concept of Internet of Things can be divided into three main elements, relating to the data that is transmitted in the system:

- data capture,

- aggregation of data,

- analysis and data processing.

The first element in the general concept of topology of IoT is data capturing. Sensors can be treated as separate components or as part of IoT devices. One device can have more than one sensor. Data obtained from the environment and pre-processed by the device can be exchanged between them in order to achieve a common goal. At this stage, the first communication occurs within the same IoT devices. It can be achieved by wireless or wired technologies.

Once all the required elements at the device level are achieved (data collection and preliminary processing), the aggregation of data starts in the IoT and the data is sent to the gateway, where later via the Internet it is delivered to different data centres. Then data is properly analyzed, processed and presented to the user with the use of appropriate applications. This processing may take place in specialized servers. It should be added that the prepared data is usually sent to the device again, so it can respond accordingly. Of course, the user can influence the IoT devices in the context of their actions (configurations).

The concept of the Internet of Things has great potential and can make life easier in many aspects. However, the creation of 
very large IoT systems may encounter many problems, which may be called the challenges which need to be addressed. The main challenge is the heterogeneity of devices. Since IoT vision involves connecting almost all devices to the Web, it has to do with the large variation mainly due to the computational and communication capabilities. In addition, this involves a huge amount of data collected from the sensors. We can talk here about the big data, that is for large and diverse data sets, where processing and analysis are difficult, but valuable, because they can lead to acquiring the new knowledge. The main element that can provide support for this challenge is well-chosen architecture and protocols [9].

Equally important challenges are still the elements associated with location, self-configuration and data management. As each device in IoT is uniquely identifiable, it is possible to locate it and its location will be used as a additional functionality. However, there is a problem with the confidentiality. Due to the number and complexity of systems, an important issue is the self-configuration, to relieve a man. It is also a challenge, since a multitude of configurations can lead to the fact that people will not understand how some elements of the system have to behaviour in the context of the property, which may prove to be as dangerous as the lack of data security. The exchange and analysis of data are important in IoT. Therefore, in addition to their safety, it is important to ensure the proper data models and description of their contents, to use them as a sure knowledge.

\section{INTERNET OF THINGS SYSTEMS USING ARTIFICIAL INTELLIGENCE}

Systems built using the concept of Internet of Things are based not only on the simple sensors that transmit information to the systems, that operate primarily on the basis of statistics and simple mathematical calculations. Such systems are increasingly complex and can make the decisions in bigger number of aspects. It is easy to imagine a system that switches the heating only based on the ambient temperature. However, it could also regulate the temperature in terms of the number of people present in the room, the habits of certain users (individuality), the specific rooms and time of a day. Therefore, to give some intelligence to these systems is an important issue, but rather complex.

Previously mentioned example with temperature and habits of users shows that IoT systems should learn the habits and adapt to them (teaching) in this case. Such elements are not achieved by ordinary statistics or simple equations. In this case the system needs more sophisticated tools, such as artificial intelligence methods.

The idea of the use of artificial intelligence in the Internet of Things is associated with another issue. It is the fact of independence of the machines in the context of their supervision. Application of AI methods can affect in a positive way to save the time. It is important not to lose a control over the device completely, but equally important is the lack of monotonous supervision of these applications from the point of use. It is better to be able to communicate with the system in a way natural for humans than for machines - based on the example with temperature - while overheating of the room, it's better to make the interaction with the device using command "is too hot" than reduce the temperature of 0.432 Celsius degree.

The main element associated with the operation of IoT system with the artificial intelligence is its location in the architecture. An important aspect is the performance and the appropriate amount of place for data, which is a knowledge of the system, so the AI methods can not be placed at each level. Figure 3 shows the general idea of placing the artificial intelligence methods in the context of major IoT architectures.

The most natural places for AI methods are all kinds of servers because of their computing power. Such location has a positive effect on another aspect too. It is about the fact of reuse. The place of operation of different artificial intelligence methods can be compared to the human brain. The fact that all the knowledge and the associated inference and learning are placed in the server rooms, it is possible to use it in a larger perspective.

Figure 4 presents our idea of general scheme of information flow in the IoT systems, using artificial intelligence. There are three main elements in the flow of information:

- Preliminary communication - data sent from the real world for a variety of systems.

- Context communication - data processed by systems that already have the appropriate context and make the IoT systems and devices can respond accordingly (take appropriate decisions).

- Internal communication - understood as an additional channel of communication between intelligent objects.

The first stage can be called a preliminary communication. At the beginning, in accordance with the concept of Internet of Things, the data from the environment (real world) is collected by the IoT devices. They can be the external sensors, as well as those built into the device. Then, data is preprocessed to be clear for the rest of the system. Depending on the particular case, the data is further transmitted over the Internet to the main IoT systems or between other devices to gather all relevant information from the real world (internal communication elements). In the context of mobile devices, it is important to assure the temporary storage of information in things during the lack of access to the Web. When all the data in the context of a particular cycle, is already in the main IoT systems, respectively, it is prepared for external artificial intelligence systems. This process may involve selecting an appropriate specific information to get to a particular method of artificial intelligence (for example the ambient temperature and the number of people as inputs to the neural network in the system controlling the temperature).

The next communication step is a context communication. Its main purpose is to provide the concrete system answers to the IoT devices and to the subsystems that are designed to take concrete actions in the context of the relevant decisions. When the artificial intelligence methods have exited and give a reply, it should be properly interpreted. This task should address the 


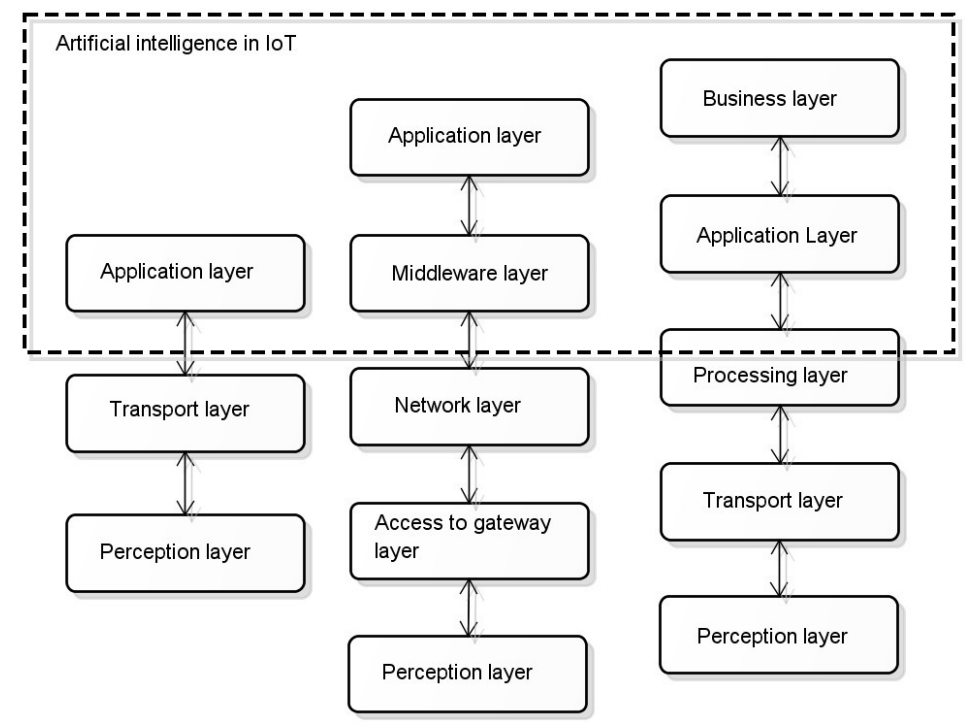

Fig. 3. Placing the artificial intelligence in the background of IoT architectures

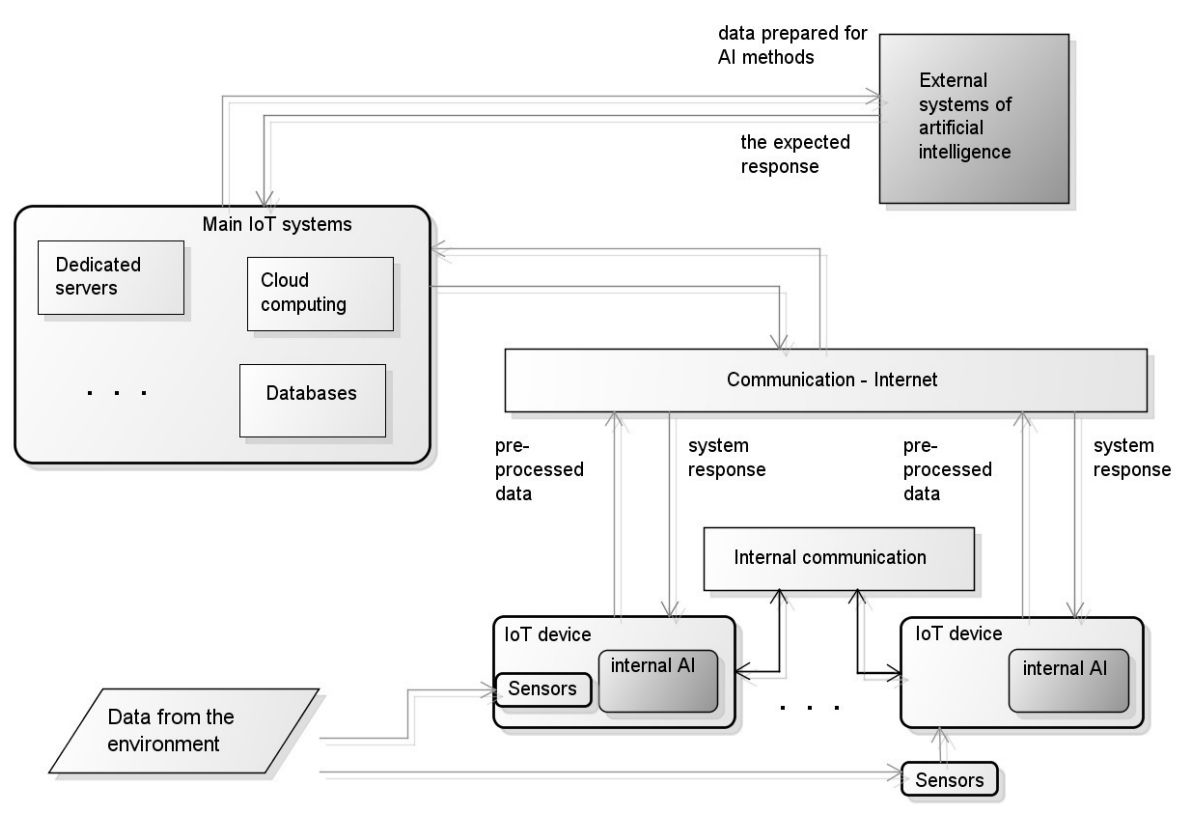

Fig. 4. General scheme of information flow in IoT systems with the use of artificial intelligence methods

main IoT systems. Very often the AI answer include digits that do not make much sense without the proper context. Therefore, an important step is to link them with the knowledge located in the system to be able to conclude the overall response of the system to a particular problem. In this way the system can properly react, so take the decision. This idea can be seen as smart making of autonomous decision.

Properly processed data, which are located in the main IoT systems should usually be further sent to certain IoT devices so that they can properly respond. A situation where the processed data must be sent to other IoT devices than those which are derived from the original data can be imagined. The monitoring system can be an example. Devices with cameras may send data to the main IoT systems, that after detecting of a specific threat (AI systems use object recognition) can send data to the other Internet of Things devices which may in some way respond to such a threat.

The last extra communication is called internal communication. It can be placed both at the initial communication, as well as at the contextual one in the proposed model. In the first case it can be used to gather enough data from multiple devices and simultaneously to send them to the upper 
layers of the system. In the second case, having a concrete answer from the system, it can be used the multiple devices work simultaneously together. This approach is combined with additional AI elements contained in the devices - it can enter into intelligent objects more aspects of their autonomy.

\section{IOT SYSTEM WITH THE USE OF ARTIFICIAL INTELLIGENCE}

To investigate the actual usefulness of selected methods of artificial intelligence in the concept of the Internet of Things, the information system smart-IoT was created. Its main task is to test the time to return home and set the alarms at the right time to put the event. The proposed system consists of three main elements:

- mobile devices - smart objects,

- central server - acts as an IoT management system,

- micro-services - include elements of AI.

The system is based on the 4-layer architecture:

- Perception layer - covers only mobile devices. Its main task is to collect the data from the environment using sensors embedded in mobile phones (GPS, accelerometer, gyroscope) and pre-treatment of this data.

- Network layer - is responsible for transferring data between all the elements of the system using LAN and Wi-Fi communications channels.

- Processing layer - an essential element of the whole system. This layer is responsible for the processing of information in a central server with the appropriate use of micro-services, so the methods of artificial intelligence. Its primary aim is to convert the input data and generate a specific answer for that data in the context of the service.

- Application layer - is responsible for providing data to the end user. Its fundamental role is to ensure the validity of data (to be updated with information from the decisionmaking module).

Intelligent objects in the proposed system are mobile devices (specifically mobile phones). Their main task is to collect the relevant data from the real world by using the built-in sensors. The next stage is the initial processing of the data. All of these tasks are performed by using the dedicated mobile application.

In accordance with the concept of Internet of Things, all devices must be uniquely identifiable throughout the system. For this reason, the smart-IoT system proposed the use of unambiguous numeric identifiers. Each mobile device has a given unique identifier, which is recorded in the central management server. Such an approach also allows to limit the access of unauthorized devices, because the system will support only those for which the information is stored in the database.

The main components of IoT, understood as the main systems of the Internet of Things, are represented by a central server, acting as a manager. It consists of 4 major components:

- module of services identification,

- module of artificial intelligence management,
- decision-making module,

- database module.

\section{CONCLUSIONS}

The Internet of Things is a technological revolution that represents the future of computing and communication. This concept is characterized by heterogeneous technologies and devices and assumes that all devices will be connected to the Internet. The next step is to add the artificial intelligence to IoT systems. Thanks to this, devices become intelligent and can make autonomous decisions. These smart devices have the ability to interact with humans and other smart devices.

These devices should have a certain autonomy in the context of decision-making process. In building of IoT systems an important element is its architecture and its scalability and flexibility. Their key action aspect is the exchange and analysis of data. Joining of this type of systems with artificial intelligence is not a trivial task. AI methods typically use a lot of processing power, therefore, using them directly in devices often becomes impossible. They are usually placed on external servers, so a user can use them in the context of multiple devices at the same time.

The presented paper investigated the usefulness of artificial intelligence in the concept of Internet of Things. To do this the exemplary system was built and it uses the artificial neural networks. This system uses mobile devices as the smart objects. Neural networks have been taught by back-propagation algorithm. Experiments with neural networks were carried out by using the main services of the system (such as auto set alarms for a specific event and estimating the time to return home). These experiments show that artificial intelligence methods are suitable for use in concept of Internet of Things.

\section{REFERENCES}

[1] A. McEwen and H. Cassimally, "Designing the Internet of Things", Wiley, 2014

[2] F. da Costa, "Rethinking the Internet of Things. A Scalable Approach to Connecting Everything", Apress open, California, 2013

[3] An Introduction to the Internet of Things (IoT), http://www. lopezresearch.com/

[4] A. Arsénio and H. Serra and R. Francisco and F. Nabais and J. Andrade and E. Serranol, "Internet of Intelligent Things: Bringing Artificial Intelligence into Things and Communication Networks", Springer Science, 2014

[5] M. Ruggieri and H. Nikookar and O. Vermesan and P. Friess, "Internet of Things: Converging Technologies for Smart Environments and Integrated Ecosystems", River Publishers, 2013

[6] D. Uckelmann and M. Harrison and F. Michahelles, "Architecting the Internet of Things", Springer, 2011

[7] M. Wu and T.-L. Lu and F.-Y. Ling and L. Sun and H.-Y. Du, "Research on the architecture of Internet of things", 3rd International Conference on Advanced Computer Theory and Engineering (ICACTE), 2010

[8] N. Lin and W. Shi, "The Research on Internet of Things Application Architecture Based on Web", IEEE Workshop on Advanced Research and Technology in Industry Applications (WARTIA), 2014

[9] C-W. Tsai and C.-F. Lai and A. V. Vasilakos, "Future Internet of Things: open issues and challenge", Springer Science, New York, 2014

[10] Cisco, http://www.cisco.com/web/about/ac79/docs/innov/IoE.pdf

[11] S. Sicari and A. Rizzardi and L. A. Grieco and A. Coen-Porisini, "Security, privacy and trust in Internet of Things: The road ahead", Computer Networks, 2014

[12] P. Lynggaard, "Artificial intelligence and Internet of Things in a "smar home" context: A Distributed System Architecture", PhD dissertation, Aalborg University Copenhagen, 2013 